\title{
ON THE NUMBER OF DISSIMILAR PFAFFIAN ORIENTATIONS OF GRAPHS *
}

\author{
Marcelo H. De Carvalho ${ }^{1}$, Cláudio L. Lucchesi ${ }^{2}$ \\ AND U.S.R. MURTY ${ }^{3}$
}

\begin{abstract}
A subgraph $H$ of a graph $G$ is conformal if $G-V(H)$ has a perfect matching. An orientation $D$ of $G$ is Pfaffian if, for every conformal even circuit $C$, the number of edges of $C$ whose directions in $D$ agree with any prescribed sense of orientation of $C$ is odd. A graph is Pfaffian if it has a Pfaffian orientation. Not every graph is Pfaffian. However, if $G$ has a Pfaffian orientation $D$, then the determinant of the adjacency matrix of $D$ is the square of the number of perfect matchings of $G$. (See the book by Lovász and Plummer [Matching Theory. Annals of Discrete Mathematics, vol. 9. Elsevier Science (1986), Chap. 8.] A matching covered graph is a nontrivial connected graph in which every edge is in some perfect matching. The study of Pfaffian orientations of graphs can be naturally reduced to matching covered graphs. The properties of matching covered graphs are thus helpful in understanding Pfaffian orientations of graphs. For example, say that two orientations of a graph are similar if one can be obtained from the other by reversing the orientations of all the edges in a cut of the graph. Using one of the theorems we proved in [M.H. de Carvalho, C.L. Lucchesi and U.S.R. Murty, Optimal ear decompositions of matching covered graphs. J. Combinat. Theory B 85 (2002) 59-93] concerning optimal ear decompositions, we show that if a matching covered graph is Pfaffian then the number of dissimilar Pfaffian orientations of $G$ is $2^{b(G)}$, where $b(G)$ is the number of "bricks" of $G$. In particular, any two Pfaffian orientations of a bipartite graph are similar. We deduce that the problem of determining whether or not a graph is Pfaffian is as difficult as
\end{abstract}

\footnotetext{
* Supported by CNPq, Brasil, by PRONEX/CNPq (664107/1997-4), by FUNDECT-MS (0284/01) and by a Fellowship for the first author from the University of Waterloo, Canada.

1 ufMS, Campo Grande, Brasil and University of Waterloo, Waterloo, Canada.

2 UNICAMP, Campinas, Brasil;lucchesi@ic.unicamp.br

3 University of Waterloo, Waterloo, Canada.
} 
the problem of determining whether or not a given orientation is Pfaffian, a result first proved by Vazirani and Yanakakis [Pfaffian orientation of graphs, 0,1 permanents, and even cycles in digraphs. Discrete Appl. Math. 25 (1989) 179-180]. We establish a simple property of minimal graphs without a Pfaffian orientation and use it to give an alternative proof of the characterization of Pfaffian bipartite graphs due to Little [A characterization of convertible (0,1)-matrices. J. Combinat. Theory B 18 (1975) 187-208].

Mathematics Subject Classification. 05C70

\section{INTRODUCTION}

Let $A=\left(a_{i j}\right)$ be an $n \times n$ skew-symmetric matrix. When $n$ is odd, then it is easy to see that $\operatorname{det}(A)=0$. On the other hand, when $n$ is even, there is a polynomial $P:=P(A)$ in the $a_{i j}$ such that $\operatorname{det} A=P^{2}$. This polynomial is called the Pfaffian of $A$ and is defined as follows:

$$
P:=\sum \operatorname{sgn}(M) a_{i_{1} j_{1}} a_{i_{2} j_{2}} \ldots a_{i_{k} j_{k}}
$$

where the sum is taken over the set of all partitions $M=\left(i_{1} j_{1}, i_{2} j_{2}, \ldots, i_{k} j_{k}\right)$ of $\{1,2, \ldots, n\}$ into $k$ unordered pairs, and $\operatorname{sgn}(M)$ is the sign of the permutation:

$$
\pi(M):=\left(\begin{array}{ccccccccc}
1 & 2 & 3 & 4 & . & . & . & 2 k-1 & k \\
i_{1} & j_{1} & i_{2} & j_{2} & . & . & . & i_{k} & j_{k}
\end{array}\right) .
$$

It can be seen that the definition of the Pfaffian of $A$ given above is independent of the order in which the constituent pairs in a partition $M$ are listed, as also of the order in which the elements in a pair are listed. Since $A$ is skew-symmetric, for each pair $(i, j)$ of indices, either $a_{i j}$ or $a_{j i}$ is nonnegative.

Now suppose that $G$ is a graph, $D$ is an orientation of $G$, and $A$ is the adjacency matrix of $D$. Then each nonzero term in the expansion of the Pfaffian of $A$ corresponds to a perfect matching $M$ of $G$. Thus, if $D$ is such that all $\operatorname{sgn}(M)$ are the same, then $|P|$ is the number of perfect matchings of $G$.

An orientation $D$ of a graph $G$ is a Pfaffian orientation of $G$ if all perfect matchings of $G$ have the same sign. (We shall see below that this is equivalent to the definition given in the abstract.) An undirected graph $G$ is Pfaffian if it admits a Pfaffian orientation.

\subsection{Parities of Circuits}

The parity of a circuit $C$ of even length in a directed graph is the parity of the number of its edges that are directed in agreement with a specified sense of orientation of $C$. As $C$ has an even number of edges, the parity is the same in both senses and thus is well defined. For any two sets $X$ and $Y$, we denote by $X \oplus Y$ the symmetric difference of $X$ and $Y$. 
Theorem 1.1 (see [12], Lem. 8.3.1). Let D be an arbitrary orientation of an undirected graph $G$. Let $M_{1}$ and $M_{2}$ be any two perfect matchings of $G$ and let $k$ denote the number of even parity circuits of $G\left[M_{1} \oplus M_{2}\right]$. Then, $M_{1}$ and $M_{2}$ have the same sign if and only if $k$ is even.

Recall that an even circuit $C$ of $G$ is conformal if $G-V(C)$ has a perfect matching. The following theorem may be derived from the above theorem.

Theorem 1.2 (see [12], Th. 8.3.2). Let $G$ be a graph, $M$ a perfect matching of $G$ and $D$ an orientation of $G$. Then the following properties are equivalent:

- $D$ is a Pfaffian orientation of $G$.

- Every $M$-alternating circuit of $G$ has odd parity.

- Every conformal circuit of $G$ has odd parity.

\subsection{Changing orientations ALONG CUtS}

For any graph $G$ and any set $X$ of vertices of $G$, we denote by $\partial(X)$ the cut of $G$ having $X$ as one of its shores, that is, the set of edges of $G$ that have precisely one end in $X$. The following simple result is fundamental for most of our results in this paper.

Proposition 1.3. In any graph $G$, a set $R$ of edges of $G$ is a cut of $G$ if and only if $|R \cap E(Q)|$ is even, for each circuit $Q$ of $G$.

If $D$ is an orientation of a graph $G$ and $S \subset E(G)$, we denote by $D \operatorname{rev}_{G} S$ the orientation of $G$ obtained from $D$ by reversing the orientation of the edges of $S$. When $G$ is understood, we only write $D \operatorname{rev} S$ instead of $D \operatorname{rev}_{G} S$. We say that two (not necessarily Pfaffian) orientations of a graph are similar if one may be obtained from the other by reversing the edges of some cut of the graph. Observe that changing the direction of an edge corresponds to changing the parity of each circuit of even length containing it. Since every circuit and every cut have an even number of edges in common, it follows that changing the orientations along a cut (that is, reversing the directions of all the arcs in a cut) preserves the parities of the circuits of even length. The following property is a direct consequence of this observation.

Proposition 1.4. Let $D$ be a Pfaffian orientation of a graph $G$. Then, any orientation of $G$ that is similar to $D$ is also Pfaffian.

One might wonder whether the converse of the above proposition is also true; that is, given two Pfaffian orientations of a graph $G$, is it possible to start from one of them and reach the other by changing orientations of the edges of some cut? In Section 3, we answer this question affirmatively for bipartite graphs. More precisely, we shall show that any two Pfaffian orientations of a bipartite graph differ by exactly a cut (that is, they are similar). In fact, we show that for any Pfaffian matching covered graph $G$, the number of dissimilar Pfaffian orientations of $G$ is equal to $2^{b(G)}$, where $b(G)$ denotes the number of bricks of $G$. 


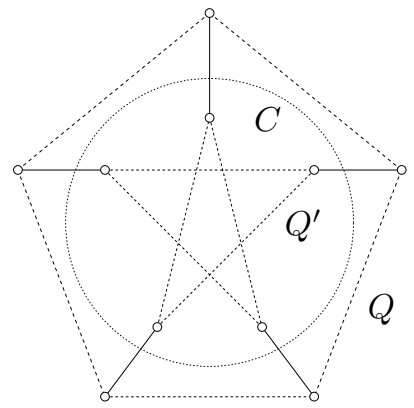

(a)

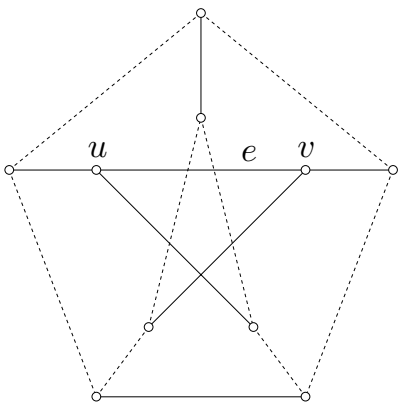

(b)

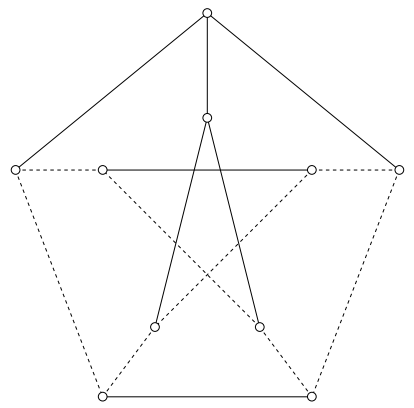

(c)

Figure 1. The Petersen graph. (a) two disjoint pentagons $Q$ and $Q^{\prime}$, and 5-cut $C$; (b) conformal octagon $R(e)$; (c) a conformal octagon containing four edges in $C$.

We remark that it follows, by Proposition 1.4, that an orientation of any spanning tree can be extended to a Pfaffian orientation of a Pfaffian graph. Using this observation, plus the fact that every circuit of $K_{3,3}$ is conformal, it is easy to prove that $K_{3,3}$ is non-Pfaffian. To illustrate the role of changing orientations along cuts in this theory, we shall now use Proposition 1.4 to show that the Petersen graph is non-Pfaffian.

We say that two orientations $D^{\prime}$ and $D^{\prime \prime}$ of an uv-path $P$ have the same parity if the parity of the number of edges directed forward coincide in $D^{\prime}$ and $D^{\prime \prime}$ when traversing $P$ from $u$ to $v$ (or from $v$ to $u$ ). The following property is easy to be verified.

Proposition 1.5. Let $P:=\left(v_{0}, v_{1}, \ldots, v_{k}\right)$ be a $v_{0} v_{k}$-path, $D_{1}$ and $D_{2}$ two orientations of $P$ that have the same parity. Then, for some subset $X$ of $\left\{v_{1}, \ldots, v_{k-1}\right\}$, $D_{2}=D_{1} \operatorname{rev} \partial(X)$.

Proposition 1.6. The Petersen graph $P$ is non-Pfaffian.

Proof. Let $Q$ be any pentagon of $P$. Then, $V(P)-V(Q)$ spans another pentagon of $P$, say $Q^{\prime}$ (see Fig. 1a).

Assume, to the contrary, that $P$ has a Pfaffian orientation. We shall now make use of Proposition 1.4 in order to show that $P$ has a Pfaffian orientation such that each of $Q$ and $Q^{\prime}$ is a directed circuit.

Indeed, given an orientation $D$ of any graph $G$ and a circuit $Q$ of $G$ of odd length, it is easy to see that there exists a set $X \subset V(Q)$ such that reversal of the edges of cut $C:=\partial(X)$ yields an orientation $D^{\prime}$ of $G$ that renders $Q$ a directed circuit. To see this, consider orientation $D$ of $G$ and note that as $Q$ has odd length, one sense of orientation of $Q$ contains an odd number of forward edges and an even number of reverse edges. Let $e$ be a forward edge of $Q, u$ and $v$ its ends. As $T:=Q-e$ is a path having an even number of forward edges in $D$, it follows by Proposition 1.5 that there exists a set $X \subset V(Q)-u-v$ such that reversal of the edges of cut $C:=\partial(X)$ in $D$ produces an orientation $D^{\prime}$ of $G$ that 
renders $T$ a directed path, in the same sense of orientation of $Q$. We conclude that $Q$ is a directed circuit in $D^{\prime}$. We remark that the shore $X$ of $C$ is a subset of $V(Q)-u-v$. Therefore, we may in fact apply this reasoning for each member of a collection of vertex-disjoint odd circuits of $G$. In particular, in the case of the Petersen graph, the pentagons $Q$ and $Q^{\prime}$ are disjoint. We may thus assume that $P$ has a Pfaffian orientation $D$ such that $Q$ and $Q^{\prime}$ are both directed circuits.

Note that $C:=\partial(V(Q))$ is a 5 -cut of $P$ (see Fig. 1a). We now show that $C$ is a directed cut in $D$. For this, observe first that for any edge $e=\{u v\}$ of $P$, $P-u-v$ contains an octagon, which is thus conformal. In particular, for each edge $e=\{u v\}$ of $Q^{\prime}, P-u-v$ contains an octagon, $R(e)$, that contains precisely two edges in $C$, plus two edges in $Q^{\prime}$, and four edges in $Q$ (see Fig. 1b). Moreover, the restriction of $R(e)$ to each of $Q$ and $Q^{\prime}$ is a directed path, or the reverse of a directed path. Since $R(e)$ must have odd parity, it follows that both edges of $R(e)$ in $C$ are directed away from $V(Q)$ or both directed away from $V\left(Q^{\prime}\right)$. This conclusion holds for each edge $e$ of $Q^{\prime}$. By applying this reasoning for any four edges $e$ of $Q^{\prime}$, we conclude that $C$ is a directed cut in $D$.

Finally, consider a conformal octagon $S$ that contains four edges in $C$ and two edges in each of $Q$ and $Q^{\prime}$ (see Fig. 1c). The edges of $S$ in $Q$ are traversed in opposite directions, relative to any sense of orientation of $Q$. Therefore, $Q$ contributes to the parity of $S$ with an odd number of forward edges. Likewise, so does $Q^{\prime}$. Finally, of the four edges of $S$ in $C$, as $C$ is directed, two are traversed in a forward manner, two in reverse manner. We deduce that $S$ has precisely four forward edges, whence it has even parity. As $S$ is conformal, this is a contradiction. As asserted, $P$ is non-Pfaffian.

The idea of using Pfaffians in matching theory is due to Tutte. In his book "Graph Theory As I Have Known It" [15], he describes how he came to the idea of using Pfaffians to find a formula for the number of perfect matchings of a graph. Although he did not succeed in finding such a formula, Tutte was able to use Pfaffian identities to prove his famous theorem characterizing graphs that have a perfect matching. This was the genesis of the theory of Pfaffian orientations. Surprisingly, the Pfaffian orientation problem is related to a number of fundamental, and seemingly unrelated, problems in graph theory. For example, the problem of deciding whether a given directed graph has an even directed circuit is equivalent to deciding if a related bipartite graph has a Pfaffian orientation [16].

Kasteleyn [8] showed that every planar graph has a Pfaffian orientation and presented a polynomial-time algorithm to find such an orientation. The graph $K_{3,3}$ is the smallest non-Pfaffian graph. Little [9] showed that a bipartite graph is non-Pfaffian if and only if it contains a conformal subgraph that is an even subdivision of $K_{3,3}$. Thus, the problem of deciding whether a given bipartite graph is Pfaffian is in $c o-\mathcal{N} \mathcal{P}$. This gave rise to the natural question whether this class of graphs is in $\mathcal{N} \mathcal{P}$. Vazirani and Yanakakis [16] showed that it is no easier to decide whether a given orientation of a graph is Pfaffian than deciding that the graph is Pfaffian and finding a Pfaffian orientation if it is. (As we shall see, for bipartite graphs this result follows from the fact that, up to similarity, there is 
only one Pfaffian orientation of a Pfaffian bipartite matching covered graph.) We give an alternate proof of this result and show that for general graphs, each of the two problems is polynomially reducible to the other. Finally, Robertson, Seymour and Thomas [14], and independently McCuaig [13], discovered a polynomial-time algorithm for deciding whether or not a bipartite graph has a Pfaffian orientation. The complexity status of the Pfaffian orientation problem for general graphs is still very much open.

\section{Matching COVERED GRAPHS}

A graph is matching covered if it has at least two vertices, is connected and each of its edges lies in a perfect matching. For instance, $K_{3,3}, K_{4}, \overline{C_{6}}$ and the Petersen graph are examples of matching covered graphs. We have seen that in the study of Pfaffian orientations we may restrict our attention to matching covered graphs. There is an extensive theory of matching covered graphs, see $[11,12]$. We shall assume that the reader is familiar with the basic concepts and results concerning matchings and matching covered graphs. However, we shall review a few basic definitions and results that are relevant to this work. For notation and terminology not defined here, see $[3,4,11]$.

\subsection{Tight CUTS}

Let $G$ be a matching covered graph. A cut $C$ is trivial if either of its shores is a singleton. A cut $C$ is odd (even) if both its shores have odd (even) cardinality. As $|V|$ is even, then every cut $C$ is either odd or even, and if $C$ is odd, then $|C \cap M|$ is odd for every perfect matching $M$ of $G$. A cut $C$ of $G$ is a tight cut if $|C \cap M|=1$ for every perfect matching $M$ of $G$. Trivial cuts are simple examples of tight cuts. The following result is a characterization of tight cuts in matching covered bipartite graphs.

Proposition 2.1. Let $G=(A, B)$ be a bipartite matching covered graph and $C$ a cut of $G$. Then, $C$ is tight if and only if there is a partition $\left(A_{1}, A_{2}\right)$ of $A$, partition $\left(B_{1}, B_{2}\right)$ of $B$, where $\left|B_{1}\right|=\left|A_{1}\right|+1$ and $\left|A_{2}\right|=\left|B_{2}\right|+1$, such that every edge of $C$ joins a vertex of $B_{1}$ to a vertex of $A_{2}$.

There are matching covered graphs which are free of nontrivial tight cuts: for example, $K_{3,3}, K_{4}, \overline{C_{6}}$ and the Petersen graph. Nonbipartite matching covered graphs free of nontrivial tight cuts are called bricks and bipartite matching covered graphs free of nontrivial tight cuts are called braces.

\subsection{Tight CUt DeCOMPosition}

Let $G$ be a connected graph and $C$ a cut of $G$. We shall refer to the graph obtained from $G$ by contracting a shore of $C$ to a single vertex as a $C$-contraction of $G$. The following property is straightforward from the definition of tight cuts. 


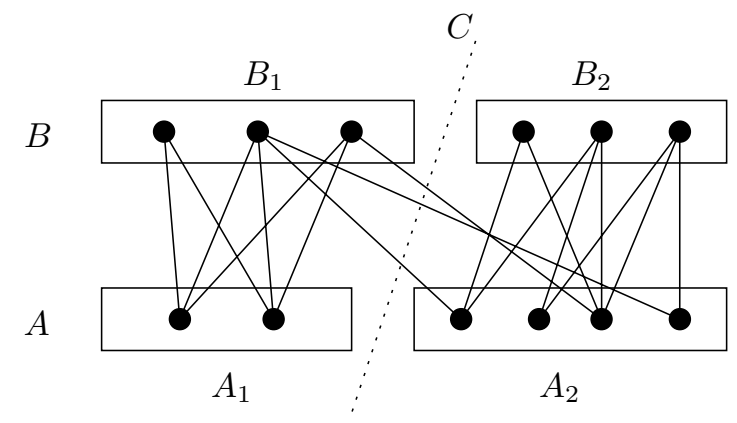

Figure 2. Tight cut in bipartite graph.

Proposition 2.2. Let $G$ be a matching covered graph, $C$ a tight cut of $G$. Then, the $C$-contractions $G_{1}$ and $G_{2}$ of $G$ are both matching covered. Moreover, for any set $R \subseteq E(G)$, if $G_{1}-R$ and $G_{2}-R$ are both matching covered then $G-R$ is also matching covered and $C-R$ is a tight cut of $G-R$.

Thus, given any matching covered graph $G$ and a nontrivial tight cut $C$ of $G$, we can obtain two smaller matching covered graphs $G_{1}$ and $G_{2}$ which are the two $C$-contractions of $G$. If either $G_{1}$ or $G_{2}$ has a nontrivial tight cut, we can take its cut-contractions, in the same manner as above, and obtain smaller matching covered graphs. Thus, by repeatedly applying cut-contractions with respect to tight cuts, we can obtain a list of graphs which do not have nontrivial tight cuts (bricks and braces). This procedure is known as tight cut decomposition.

Theorem 2.3 (see [11]). Any two applications of the tight cut decomposition procedure on a matching covered graph $G$ produce the same list of bricks and braces, except possibly for the multiplicities of edges.

In particular, the numbers of bricks and braces resulting from a tight cut decomposition of a matching covered graph $G$ is independent of the tight cut decomposition; we shall call these the numbers of bricks and braces of $G$ respectively. We shall let $b(G)$ denote the number of bricks of $G$, and $p(G)$ denote the number of bricks whose underlying simple graphs are Petersen graphs. The number $b(G)$ plays an important role in this paper. We remark that in view of Proposition 2.1, if $G$ is a bipartite matching covered graph then $b(G)=0$.

The next result shows the importance of tight cuts in the study of Pfaffian orientations.

Theorem 2.4 (see [10]). Let $G$ be a matching covered graph and $C:=\partial(X)$ a tight cut of $G$. Then $G$ is Pfaffian if and only if the two $C$-contractions of $G$ are Pfaffian.

It follows that once we have characterized Pfaffian bricks and braces, we have characterized all Pfaffian graphs. Thus, in the study of the Pfaffian orientation problem, we may restrict our attention to bricks and braces. Another important 
remark is that by Theorem 2.4 and Proposition 1.6, if a matching covered graph $G$ has $p(G)>0$ then $G$ is non-Pfaffian.

\subsection{MinorS}

Recall that a subgraph $H$ of a graph $G$ is a conformal subgraph of $G$ if $G-V(H)$ has a perfect matching. The property of being a conformal subgraph is transitive, that is, if $G^{\prime \prime}$ is a conformal subgraph of $G^{\prime}$ and $G^{\prime}$ is a conformal subgraph of $G$ then $G^{\prime \prime}$ is also a conformal subgraph of $G$. Observe that a graph is a conformal subgraph of itself. By Theorem 1.2, we immediately deduce that a graph $G$ is Pfaffian if and only if every conformal subgraph of $G$ is Pfaffian.

Let $G$ be a matching covered graph, let $u$ and $v$ be two adjacent vertices of degree two in $G$, and let $w$ be the neighbor of $v$ different from $u$. Then $\partial(X)$, where $X:=\{u, v, w\}$, is a tight cut of $G$. One of the $\partial(X)$-contractions is a brace on four vertices; let $H$ denote the other $\partial(X)$-contraction. Since a brace on four vertices is Pfaffian, it follows from Theorem 2.4 that $G$ is Pfaffian if and only if $H$ is Pfaffian. A graph $G$ is an even subdivision of a graph $H$ if $G$ can be obtained from $H$ by even subdivisions of its edges. It follows from the above observation that a graph is Pfaffian if and only if each of its even subdivisions is Pfaffian.

A graph $H$ is a conformal minor (or simply a minor) of $G$ if there exists an even subdivision of $H$ that is a conformal subgraph of $G$. From the above observations one can deduce directly that:

Proposition 2.5. A graph $G$ is Pfaffian if and only if every minor of $G$ is Pfaffian.

Little [9] proved that a bipartite matching covered graph is Pfaffian if and only if it does not contain $K_{3,3}$ as a minor. In Section 4 we present an alternative proof of this result.

\subsection{EAR DECOMPOSITIONS}

Let $G$ be a connected graph. A single ear of $G$ is a path $P$ of odd length in $G$ whose internal vertices (if any) have degree two in $G$. If $P$ is a single ear of $G$ then we denote by $G-P$ the graph obtained from $G$ by deleting the edges and internal vertices of $P$. The following theorem provides a decomposition of bipartite matching covered graphs.

Theorem 2.6 (see[12], Th. 4.1.6). Given any bipartite matching covered graph $G$, there exists a sequence

$$
G_{1} \subset G_{2} \subset \ldots \subset G_{r}=G
$$

of conformal matching covered subgraphs of $G$ where (i) $G_{1}=K_{2}$, and (ii) for $2 \leq i \leq r, G_{i-1}=G_{i}-R_{i}$, where $R_{i}$ is a single ear of $G_{i}$.

Such decompositions do not exist for non-bipartite matching covered graphs. For example, $K_{4}$ and $\overline{C_{6}}$ have no ear decomposition as in Theorem 2.6. However, every matching covered graph has an ear decomposition with a slight relaxation 
of the above definition. To describe that decomposition, we require the notion of a double ear.

A double ear of $G$ is a pair $\left(R_{1}, R_{2}\right)$, where $R_{1}$ and $R_{2}$ are two vertex-disjoint single ears of $G$. An ear of $G$ is either a single ear or a double ear of $G$. If $R$ is an ear of $G$ then we denote by $G-R$ the graph obtained from $G$ by deleting the edges and internal vertices of the constituent paths of $R$.

A single ear $R$ of $G$ is removable if the graph $G-R$ is matching covered. A removable single ear of length one is a removable edge. A double ear $R=\left(R_{1}, R_{2}\right)$ of $G$ is removable if $G-R$ is matching covered. In this case, it is to be understood that neither $R_{1}$ nor $R_{2}$ is a removable single ear. A removable ear of $G$ is either a single or a double ear which is removable. A removable double ear in which both constituent paths have length one is a removable doubleton. Observe that a removable ear of a brick is either a singleton or a doubleton.

An ear decomposition of a matching covered graph $G$ is a sequence

$$
G_{1} \subset G_{2} \subset \ldots \subset G_{r}=G
$$

of subgraphs of $G$ where (i) $G_{1}=K_{2}$, and (ii) for $2 \leq i \leq r, G_{i-1}=G_{i}-R_{i}$, where $R_{i}$ is a removable ear of $G_{i}$. Note that each term of an ear decomposition of $G$ is a conformal matching covered subgraph of $G$. The following fundamental theorem was established by Lovász and Plummer.

Theorem 2.7 (the two-ear theorem [12], Th. 5.4.2). Every matching covered graph has an ear decomposition.

A removable ear $R$ of a matching covered graph $G$ is $b$-removable if either (i) $R$ is a single ear and $b(G-R)=b(G)$ or (ii) $R$ is a double ear and $b(G-R)=b(G)-1$. An ear decomposition of a matching covered graph is optimal if it uses the least possible number of double ears. In [5], Theorem 1.3, we proved that in every matching covered graph $G$ the optimal number of double ears is $b(G)+p(G)$. For this we used a result [5], Theorem 6.2, that implies the following statement:

Theorem 2.8. Every matching covered graph distinct from $K_{2}$ and such that $p(G)=0$ contains a $b$-removable ear.

\section{Number OF Dissimilar PfAFFiAn ORIENTATIONS}

We say that two orientations $D^{\prime}$ and $D^{\prime \prime}$ of a graph $G$ are similar if there is a cut $C$ such that $D^{\prime \prime}=D^{\prime}$ rev $C$. We start with a very simple observation.

Proposition 3.1. Similarity is an equivalence relation on the set of all orientations of a graph.

\subsection{A LOWER Bound on the NUmber of dissimilar Pfaffian orientations OF MATCHING COVERED GRAPHS}

Let $G$ be a matching covered graph and let $D$ be a Pfaffian orientation of $G$. We shall proceed to describe how to obtain $2^{b(G)}$ Pfaffian reorientations of $D$, no two 
of which are similar. For this purpose, we consider a tight cut decomposition $\mathcal{G}$ of $G$. Given any subset $\mathcal{B}$ of bricks in $\mathcal{G}$, we shall see that the directed graph obtained by reversing the orientations of all the arcs in $R:=\oplus_{B \in \mathcal{B}} E(B)$ is also a Pfaffian orientation of $G$. The difficult part is to show that distinct subsets of bricks give rise to dissimilar Pfaffian orientations. The following lemma will be used in establishing that fact.

Lemma 3.2. Let $G$ be a matching covered graph, $\mathcal{G}$ a tight cut decomposition of $G, \mathcal{B}$ a subset of $\mathcal{G}, R:=\oplus_{B \in \mathcal{B}} E(B)$. The following properties hold:

(i) for each conformal circuit $Q$ of $G,|R \cap E(Q)|$ is even;

(ii) set $R$ is a cut if and only if $\mathcal{B}$ consists solely of braces.

Proof. By induction on $|V(G)|$. If $\mathcal{B}$ is empty then the assertion holds immediately. We may thus assume that $\mathcal{B}$ contains at least one brick or one brace.

Consider first the case in which $G$ is either a brick or a brace, in which case $G$ is the only element of $\mathcal{B}$. Then, $R=E(G)$. Every conformal circuit $Q$ of $G$ has even length, whence $|R \cap E(Q)|$ is even. By Proposition 1.3, set $R$ is a cut if and only if $|R \cap E(Q)|$ is even, for every circuit $Q$ of $G$. That is, $R$ is a cut of $G$ if and only if $G$ is bipartite. The assertion holds if $G$ is a brick or a brace.

We may thus assume that $G$ has nontrivial tight cuts. Let $C$ be a nontrivial tight cut of $G$ that is used to produce $\mathcal{G}$. Let $G_{1}$ and $G_{2}$ denote the two $C$ contractions of $G$. For $i=1,2$, let $\mathcal{G}_{i}$ and $\mathcal{B}_{i}$ denote the restrictions of $\mathcal{G}$ and $\mathcal{B}$, respectively, to bricks and braces of $G_{i}$, let $R_{i}:=\oplus_{B \in \mathcal{B}_{i}} E(B)$. Then, $\left\{\mathcal{G}_{1}, \mathcal{G}_{2}\right\}$ is a partition of $\mathcal{G},\left\{\mathcal{B}_{1}, \mathcal{B}_{2}\right\}$ is a partition of $\mathcal{B}, \mathcal{G}_{i}$ is a tight cut decomposition of $G_{i}$ and $\mathcal{B}_{i} \subseteq \mathcal{G}_{i}$, for $i=1,2$. Moreover, $R=R_{1} \oplus R_{2}$.

To prove part (i) of the assertion, let $Q$ be a conformal circuit of $G$. Consider first the case in which $E(Q)$ and $C$ are disjoint. As $C$ is tight in $G$, it follows that $Q$ is a conformal circuit of one of $G_{1}$ and $G_{2}$. Adjust notation so that $Q$ is a conformal circuit of $G_{1}$. Then, $R \cap E(Q)=R_{1} \cap E(Q)$. By induction hypothesis, $\left|R_{1} \cap E(Q)\right|$ is even. Thus, $|R \cap E(Q)|$ is even. Part (i) of the assertion holds in this case.

Consider next the case in which $Q$ has at least one edge in $C$. As $C$ is tight in $G$, circuit $Q$ contains precisely two edges in $C$. For $i=1,2$, let $Q_{i}$ denote the circuit of $G_{i}$ spanned by $E(Q) \cap E\left(G_{i}\right)$. Then, $Q_{i}$ is a conformal circuit of $G_{i}$. By induction hypothesis, $\left|R_{i} \cap E\left(Q_{i}\right)\right|$ is even. Thus,

$$
R \cap E(Q)=\left(R_{1} \oplus R_{2}\right) \cap E(Q)=\left(R_{1} \cap E\left(Q_{1}\right)\right) \oplus\left(R_{2} \cap E\left(Q_{2}\right)\right) .
$$

By induction hypothesis, each of $\left|R_{1} \cap E\left(Q_{1}\right)\right|$ and $\left|R_{2} \cap E\left(Q_{2}\right)\right|$ is even, thus $|R \cap E(Q)|$ is also even. The proof of (i) is complete.

To prove part (ii) of the assertion, consider first the case in which $\mathcal{B}$ consists solely of braces. Then, each graph in $\mathcal{B}_{1}$ is a brace. By induction hypothesis, $R_{1}$ is a cut of $G_{1}$. Thus, $R_{1}$ is a cut of $G$. Let $X_{1}$ be a shore of $R_{1}$ in $G$. Likewise, $R_{2}$ is a cut of $G$, let $X_{2}$ be a shore of $R_{2}$ in $G$. Then, $R=R_{1} \oplus R_{2}=\partial_{G}\left(X_{1} \oplus X_{2}\right)$ is a cut of $G$. Part (ii) of the assertion holds if each graph in $\mathcal{B}$ is a brace. 
Consider last the case in which $\mathcal{B}$ contains at least one brick. Then, at least one of $\mathcal{B}_{1}$ and $\mathcal{B}_{2}$ contains at least one brick. Adjust notation so that $\mathcal{B}_{1}$ contains at least one brick. By induction hypothesis, $R_{1}$ is not a cut of $G_{1}$. By Proposition 1.3, $G_{1}$ contains a circuit, $Q_{1}$, such that $\left|R_{1} \cap E\left(Q_{1}\right)\right|$ is odd. If $Q_{1}$ contains no edges in $C$ then $Q_{1}$ is a circuit of $G$ and $R \cap E\left(Q_{1}\right)=R_{1} \cap E\left(Q_{1}\right)$, whence $\left|R \cap E\left(Q_{1}\right)\right|$ is odd. By Proposition 1.3, set $R$ is not a cut of $G$.

We may thus assume that $Q_{1}$ has (precisely two) edges in $C$, say $e$ and $f$. Let $M_{e}$ and $M_{f}$ denote two perfect matchings of $G$ that contain edges $e$ and $f$, respectively. As $C$ is tight, edge $e$ does not lie in $M_{f}$ and $f$ does not lie in $M_{e}$. Thus, the $M_{e}, M_{f}$-alternating circuit $S$ of $G$ that contains edge $e$ contains also edge $f$ and no other edge of $C$. The set $E\left(G_{2}\right) \cap E(S)$ spans in $G_{2}$ a circuit, say $Q_{2}$, that is conformal in $G_{2}$. Moreover, $E\left(Q_{1}\right) \cup E\left(Q_{2}\right)$ spans in $G$ a circuit, $Q$. Then,

$$
R \cap E(Q)=\left(R_{1} \oplus R_{2}\right) \cap E(Q)=\left(R_{1} \cap E\left(Q_{1}\right)\right) \oplus\left(R_{2} \cap E\left(Q_{2}\right)\right) .
$$

Note that $R_{2} \cap E\left(Q_{2}\right)=R_{2} \cap E(S)$. By part (i), with $\mathcal{B}_{2}$ playing the role of $\mathcal{B}$, and since $S$ is a conformal circuit of $G$, it follows that $\left|R_{2} \cap E\left(Q_{2}\right)\right|$ is even. By defnition of $Q_{1},\left|R_{1} \cap E\left(Q_{1}\right)\right|$ is odd. It follows that $|R \cap E(Q)|$ is odd. By Proposition 1.3, set $R$ is not a cut of $G$. The proof of (ii) completes the proof of the Lemma.

Corollary 3.3. Every Pfaffian matching covered graph $G$ has at least $2^{b(G)}$ dissimilar Pfaffian orientations.

Proof. By hypothesis, $G$ has a Pfaffian orientation, say $D$. Let $\mathcal{G}_{0}$ be the collection of (precisely $b(G)$ ) bricks of a tight cut decomposition of $G$. For each subset $\mathcal{B}$ of $\mathcal{G}_{0}$, let $R(\mathcal{B}):=\oplus_{B \in \mathcal{B}} E(B)$, let $D(\mathcal{B}):=D$ rev $R(\mathcal{B})$. Let $\mathcal{D}:=\left\{D(\mathcal{B}): B \subseteq \mathcal{G}_{0}\right\}$.

By part (i) of the lemma and Theorem 1.2, the $2^{b(G)}$ orientations in $\mathcal{D}$ are Pfaffian. To complete the proof, we now show that they are dissimilar. For this, let $\mathcal{B}_{1}$ and $\mathcal{B}_{2}$ denote two distinct subsets of $\mathcal{G}_{0}$. Then, $\mathcal{B}:=\mathcal{B}_{1} \oplus \mathcal{B}_{2}$ is a nonnull subset of $\mathcal{G}_{0}$. Moreover, $R(\mathcal{B})=R\left(\mathcal{B}_{1}\right) \oplus R\left(\mathcal{B}_{2}\right)$, whence $D\left(\mathcal{B}_{2}\right)=D\left(\mathcal{B}_{1}\right) \operatorname{rev} R(\mathcal{B})$. By the Lemma, part (ii), $R(\mathcal{B})$ is not a cut of $G$. Therefore, $D\left(\mathcal{B}_{1}\right)$ and $D\left(\mathcal{B}_{2}\right)$ are dissimilar. This conclusion is valid for any two distinct subsets $\mathcal{B}_{1}$ and $\mathcal{B}_{2}$ of $\mathcal{G}_{0}$. The assertion holds.

\subsection{Pfaffian extensions}

In this section we use Theorem 2.8 in order to prove that every Pfaffian matching covered graph $G$ has precisely $2^{b(G)}$ dissimilar Pfaffian orientations. We also show how to determine Pfaffian orientations for Pfaffian matching covered graphs.

Theorem 3.4. Let $G$ be a matching covered graph, $\mathcal{D}$ a collection of $2^{b(G)}$ dissimilar Pfaffian orientations of $G$. Let $R$ be a b-removable ear of $G, Q$ a conformal circuit of $G$ that contains some edge of $R, D_{0}$ an orientation of $G$ such that (i) $D_{0}-R$ is a Pfaffian orientation of $G-R$ and (ii) $Q$ has odd parity in $D_{0}$. If $G-R$ has precisely $2^{b(G-R)}$ dissimilar Pfaffian orientations then $D_{0}$ is similar to some orientation in $\mathcal{D}$. 
Proof. Let $G^{\prime}:=G-R, t:=2^{b(G)}$. Denote by $D_{1}, D_{2}, \ldots, D_{t}$ the $t$ dissimilar Pfaffian orientations in $\mathcal{D}$. For $i=0,1, \ldots, t$, let $D_{i}^{\prime}:=D_{i}-R$. For $i>0, D_{i}^{\prime}$ is a Pfaffian orientation of $G^{\prime}$, because $D_{i}$ is a Pfaffian orientation of $G$. Moreover, $D_{0}^{\prime}$ is a Pfaffian orientation of $G^{\prime}$, by hypothesis.

Consider first the case in which $R$ is a single ear. In that case, $b\left(G^{\prime}\right)=b(G)$. By hypothesis, $G^{\prime}$ has precisely $t$ dissimilar Pfaffian orientations. Therefore, there are indices $i$ and $j$ such that $D_{i}^{\prime}$ and $D_{j}^{\prime}$ are similar, where $0 \leq i<j \leq t$. Let $C^{\prime}$ denote a cut of $G^{\prime}$ such that $D_{i}^{\prime}=D_{j}^{\prime}$ rev $C^{\prime}$. Let $X$ denote a shore of $C^{\prime}$ in $G^{\prime}$. Let $C:=\partial_{G}(X)$. Then, $D_{j}^{\star}:=D_{j} \operatorname{rev} C$ is similar to $D_{j}$, whence $D_{j}^{\star}$ is a Pfaffian orientation of $G$. Moreover, for each edge of $G^{\prime}$, its orientations in $D_{i}$ and in $D_{j}^{\star}$ coincide. If $i=0$ then $Q$ has odd parity in $D_{i}$, by hypothesis. If $i>0$ then $Q$ has odd parity in $D_{i}$, because $D_{i}$ is Pfaffian. As $D_{j}^{\star}$ is Pfaffian, $Q$ has odd parity in $D_{j}^{\star}$. As $R$ is a single ear of $G$ and $Q$ contains an edge of $R$, it contains all the edges of $R$. Each edge of $E(Q)-E(R)$ has the same orientation in $D_{i}$ and in $D_{j}^{\star}$. We conclude that $R$ has the same parity in $D_{i}$ and in $D_{j}^{\star}$. By Proposition $1.5, D_{i}$ and $D_{j}^{\star}$ are similar. Thus, $D_{i}$ and $D_{j}$ are similar. As the orientations in $\mathcal{D}$ are dissimilar, it follows that $i=0$. The assertion holds if $R$ is a single ear.

Consider next the case in which $R$ is a double ear. In that case, $b\left(G^{\prime}\right)=b(G)-1$. By hypothesis, $G^{\prime}$ has precisely $t / 2$ dissimilar Pfaffian orientations. Therefore, there are indices $i, j$ and $k$ such that $D_{i}^{\prime}, D_{j}^{\prime}$ and $D_{k}^{\prime}$ are similar, where $0 \leq i<$ $j<k \leq t$. Let $C_{j}^{\prime}$ and $C_{k}^{\prime}$ denote cuts of $G^{\prime}$ such that $D_{i}^{\prime}=D_{j}^{\prime} \operatorname{rev} C_{j}^{\prime}=D_{k}^{\prime} \operatorname{rev} C_{k}^{\prime}$. Let $X_{j}$ and $X_{k}$ denote shores of $C_{j}^{\prime}$ and $C_{k}^{\prime}$ in $G^{\prime}$, respectively. Let $C_{j}:=\partial_{G}\left(X_{j}\right)$, $C_{k}:=\partial_{G}\left(X_{k}\right)$. Then, $D_{j}^{\star}:=D_{j} \operatorname{rev} C_{j}$ is similar to $D_{j}$, whence $D_{j}^{\star}$ is a Pfaffian orientation of $G$. Likewise, $D_{k}^{\star}:=D_{k}$ rev $C_{k}$ is similar to $D_{k}$, whence $D_{k}^{\star}$ is a Pfaffian orientation of $G$. Moreover, for each edge of $G^{\prime}$, its orientations in $D_{i}$, $D_{j}^{\star}$ and in $D_{k}^{\star}$ coincide. If $i=0$ then $Q$ has odd parity in $D_{i}$, by hypothesis. If $i>0$ then $Q$ has odd parity in $D_{i}$, because $D_{i}$ is Pfaffian. As $D_{j}^{\star}$ and $D_{k}^{\star}$ are both Pfaffian, $Q$ has odd parity in both. Let $R_{1}$ and $R_{2}$ denote the two constituent paths of $R$. Adjust notation so that $Q$ contains an edge of $R_{1}$. Then, $Q$ contains all the edges of $R_{1}$. If $Q$ does not contain any edge in $R_{2}$ then $G-R_{2}$ is matching covered, whence $R_{2}$ is a removable ear of $G$, a contradiction to the definition of removable double ear. We conclude that $Q$ has edges in $R_{2}$, whence it contains all the edges of $R_{2}$. It follows that $Q$ contains all the edges of $R$. Path $R_{1}$ has the same parity in at least two of $D_{i}, D_{j}^{\star}$ and $D_{k}^{\star}$. As each edge of $E(Q)-E(R)$ has the same orientation in those three orientations, and since $Q$ has odd parity in those three orientations, it follows that in at least two of the orientations $D_{i}, D_{j}^{\star}$ and $D_{k}^{\star}$, each of $R_{1}$ and $R_{2}$ has the same parity. By Proposition 1.5, at least two of $D_{i}, D_{j}^{\star}$ and $D_{k}^{\star}$ are similar. Thus, at least two of $D_{i}, D_{j}$ and $D_{k}$ are similar. As the orientations in $\mathcal{D}$ are dissimilar, it follows that $i=0$ and $D_{i}$ is similar to (precisely) one of $D_{j}$ and $D_{k}$. The assertion holds if $R$ is a double ear.

Corollary 3.5. Every Pfaffian matching covered graph $G$ has precisely $2^{b(G)}$ dissimilar Pfaffian orientations.

Proof. By induction on $|E(G)|$. Let $t:=2^{b(G)}$. By hypothesis, $G$ is Pfaffian. By Corollary 3.3, $G$ has a collection $\mathcal{D}$ of $t$ dissimilar Pfaffian orientations. To prove 
the assertion, we now show that every Pfaffian orientation $D$ of $G$ is similar to some orientation in $\mathcal{D}$.

The assertion holds immediately if $G$ is $K_{2}$. We may thus assume that $G$ is distinct from $K_{2}$. As $G$ is Pfaffian, we have that $p(G)=0$. By Theorem 2.8, $G$ has a $b$-removable ear $R$. As $D$ is Pfaffian, $D-R$ is a Pfaffian orientation of $G-R$. Every conformal circuit of $G$ has odd parity in $D$. By induction hypothesis, $G-R$ has precisely $2^{b(G-R)}$ dissimilar Pfaffian orientations. By Theorem 3.4, $D$ is similar to some orientation in $\mathcal{D}$. This conclusion holds for each Pfaffian orientation $D$ of $G$.

Corollary 3.6. Let $G$ be a matching covered graph, $R$ a b-removable ear of $G$, $D$ an orientation of $G, Q$ a conformal circuit of $G$ that contains some edge of $R$. Assume that (i) $D-R$ is a Pfaffian orientation of $G-R$ and (ii) $Q$ has odd parity in $D$. Then, $G$ is Pfaffian if and only if $D$ is a Pfaffian orientation of $G$.

Proof. If $D$ is a Pfaffian orientation of $G$ then $G$ is certainly Pfaffian. To prove the converse, assume that $G$ is Pfaffian. By Corollary $3.3, G$ has a collection $\mathcal{D}$ of $2^{b(G)}$ dissimilar Pfaffian orientations. By Corollary 3.5, $G-R$ has precisely $2^{b(G-R)}$ dissimilar Pfaffian orientations. By Theorem 3.4, $D$ is similar to some orientation in $\mathcal{D}$. Thus, $D$ is a Pfaffian orientation of $G$.

\subsection{Polynomial Reducibilities}

In this section we give a polynomial algorithm that, given a matching covered graph $G$, produces an orientation $D$ of $G$ such that $G$ is Pfaffian if and only if $D$ is Pfaffian. We also give polynomial reductions relating two problems: (i) to determine whether or not a given matching covered graph is Pfaffian and (ii) to determine whether or not a given orientation of a matching covered graph is Pfaffian. We need to record first some facts concerning the complexity of some problems related to matching covered graphs.

The literature has several polynomial algorithms to determine, given a graph $G$, a perfect matching of $G$, or alternatively, a certificate that $G$ has no perfect matchings; the certificate is a set $S$ of vertices of $G$ such that the number of odd components of $G-S$ is strictly larger than $|S|$. Using any such algorithm, it is possible to determine in polynomial time whether or not a given graph $G$ is matching covered. In linear time it is possible to verify whether or not $G$ is connected. For each edge $e$ of $G$, if $u$ and $v$ denote the ends of $e, G$ has a perfect matching that contains edge $e$ if and only if graph $G-u-v$ has a perfect matching.

We remark that to determine, in polynomial time, a conformal circuit of a given matching covered graph $G$ distinct from $K_{2}$ that contains a given edge $e$ of $G$, it suffices to determine a perfect matching $M$ of $G$ that contains edge $e$ and a perfect matching $N$ of $G$ that does not contain edge $e$, and then take the $M, N$-alternating circuit of $G$ that contains edge $e$.

To determine the set of removable ears of a matching covered graph $G$ in polynomial time, let $\mathcal{P}$ denote the set of paths $P$ of $G$ of maximum length such that each internal vertex of $P$ has degree two. If $G$ is not a circuit, then $\mathcal{P}$ induces a 
partition of $E(G)$ and $\mathcal{P}$ may be determined in linear time. The set of removable ears of an even circuit is trivially determined, so assume that $G$ is not a circuit. Every constituent path of a removable ear of $G$ lies in $\mathcal{P}$ and has odd length. Thus, the set of removable single ears of $G$ is the set of those paths $R$ in $\mathcal{P}$ that have odd length and such that $G-R$ is matching covered. Likewise, the set of removable double ears of $G$ is the set of those vertex-disjoint pairs $\left\{R_{1}, R_{2}\right\}$ of paths in $\mathcal{P}$ such that each of $R_{1}$ and $R_{2}$ has odd length, $G-R_{1}-R_{2}$ is matching covered and neither $R_{1}$ nor $R_{2}$ is removable.

We now make some remarks concerning the determination, in polynomial time, of the number $b(G)$ of bricks of any tight cut decomposition of $G$. In linear time, determine whether or not $G$ is bipartite. If $G$ is bipartite then $b(G)=0$. Assume thus that $G$ is nonbipartite. For each pair $\{u, v\}$ of distinct vertices $u$ and $v$ of $G$, determine whether or not $G-u-v$ is connected and has a perfect matching. If, for some such pair, $G-u-v$ has no perfect matching, then let $B:=S \cup\{u, v\}$, where $S$ is a certificate of the nonexistence of a perfect matching in $G-u-v$. As $G$ is matching covered, it follows that the number of odd components of $G-B$ is equal to $|B|$, no edge of $G$ has both ends in $B$ and each component of $G-B$ is odd. As $G$ is nonbipartite, at least one (odd) component of $G-B$, say $K$, is nontrivial. In that case, $C:=\partial(V(K))$ is a nontrivial tight cut of $G$. Then, $b(G)=b\left(G_{1}\right)+b\left(G_{2}\right)$, where $G_{1}$ and $G_{2}$ are the two $C$-contractions of $G$, the algorithm procedes recursively. Assume thus that $G-u-v$ has a perfect matching, for each pair $\{u, v\}$ of distinct vertices of $G$. Then, $G$ is said to be bicritical. We thus have a polynomial algorithm to determine whether or not a matching covered graph is bicritical.

We may assume that $G$ is bicritical. If, for some pair $\{u, v\}, G-u-v$ is not connected, then, as $G$ is bicritical, each component of $G-u-v$ is even. For any component $K$ of $G-u-v, C:=\partial(V(K) \cup\{u\})$ is a nontrivial tight cut of $G$. Again, recursively determine $b\left(G_{1}\right)$ and $b\left(G_{2}\right)$, where $G_{1}$ and $G_{2}$ are the $C$-contractions of $G$ and determine $b(G)=b\left(G_{1}\right)+b\left(G_{2}\right)$.

Finally, if $G$ is bicritical and 3-connected, then $G$ is a brick, by the following assertion, proved by Edmonds, Lovász and Pulleyblank [6].

Theorem 3.7. A nonbipartite matching covered graph $G$ is a brick if and only if it is 3-connected and bicritical.

In that case, $b(G)=1$. We have thus very briefly described a polynomial algorithm for determining $b(G)$ for a given matching covered graph $G$.

We end these preliminary remarks with the following observation. In polynomial time, we know how to determine the set of removable ears of $G$ and also, for each such ear $R$, the numbers $b(G)$ and $b(G-R)$. Therefore, in polynomial time we know how to determine the set of $b$-removable ears of $G$.

Lemma 3.8. There is a polynomial algorithm that, given a matching covered graph $G$, either determines (i) a conformal subgraph $H$ of $G$ such that $p(H)>0$, or (ii) an ear decomposition

$$
G_{1} \subset G_{2} \subset \ldots \subset G_{r}=G
$$

of $G$ where for $2 \leq i \leq r, G_{i-1}=G_{i}-R_{i}$, and $R_{i}$ is a b-removable ear of $G_{i}$. 
Proof. If $G$ is $K_{2}$ then $r:=1$ and $G_{1}:=G$. Assume thus that $G$ is not $K_{2}$. Determine, in polynomial time, a $b$-removable ear of $G$, if one exists. By Theorem 2.8, if no such ear exists then $p(G)>0$ and we are done. If a $b$-removable ear $R$ is found, then recursively run the algorithm for matching covered graph $G-R$. Any conformal subgraph of $G-R$ is a conformal subgraph of $G$. If $G-R$ has a conformal subgraph $H$ such that $p(H)>0$, then $H$ is a conformal subgraph of $G$ and we are done. Alternatively, an ear decomposition

$$
G_{1} \subset G_{2} \subset \ldots \subset G_{r-1}=G-R
$$

is obtained, where for each $i, 2 \leq i<r, G_{i-1}=G_{i}-R_{i}$, and $R_{i}$ is a $b$-removable ear of $G_{i}$. Add to that decomposition graph $G_{r}:=G$ as its last term, where $R_{r}:=R$ is the $b$-removable ear of $G_{r}$ such that $G_{r-1}=G_{r}-R_{r}$.

Theorem 3.9. There exists a polynomial algorithm that, given a matching covered graph $G$, determines an orientation $D$ of $G$ such that $G$ is Pfaffian if and only if $D$ is a Pfaffian orientation of $G$.

Proof. Run the algorithm described in the proof of Lemma 3.8. We now define the orientation $D$ as follows. If a conformal subgraph $H$ of $G$ such that $p(H)>0$ has been obtained, then $G$ is not Pfaffian, and any orientation $D$ of $G$ satisfies the assertion. We may thus assume that an ear decomposition

$$
G_{1} \subset G_{2} \subset \ldots \subset G_{r}=G
$$

was determined, where, for $i=2,3, \ldots, r, G_{i-1}=G_{i}-R_{i}$, and $R_{i}$ is a $b$-removable ear of $G$. Orient $G_{1}$ arbitrarily. For $i=2,3, \ldots, r$, extend the orientation $D_{i-1}$ of $G_{i-1}$ to an orientation $D_{i}$ of $G_{i}$ as follows: determine in polynomial time a conformal circuit $Q_{i}$ of $G_{i}$ that contains some edge in $R_{i}$. Orient the edges of $R_{i}$ so that $Q_{i}$ has odd parity in $D_{i}$.

We now show, by induction on $i$, that $G_{i}$ is Pfaffian if and only if $D_{i}$ is a Pfaffian orientation of $G_{i}$. This assertion holds immediately for $i=1$. Assume thus that $i>1$. If $D_{i}$ is a Pfaffian orientation of $G_{i}$, then $G_{i}$ is certainly Pfaffian. To prove the converse, assume that $G_{i}$ is Pfaffian. Then, $G_{i-1}$ is Pfaffian. By induction, $D_{i-1}$ is a Pfaffian orientation of $G_{i-1}$. By Corollary 3.6, it follows that $D_{i}$ is a Pfaffian orientation of $G_{i}$. In particular, $G$ is Pfaffian if and only if $D:=D_{r}$ is a Pfaffian orientation of $G$.

With the next two corollaries we establish the result first proved by Vazirani and Yanakakis [16], that it is no easier to decide whether a given orientation of a graph is Pfaffian than deciding that the graph is Pfaffian and finding a Pfaffian orientation if it is.

Corollary 3.10. The problem of determining whether or not a given matching covered graph is Pfaffian is polynomially reducible to the problem of deciding whether or not a given orientation of a matching covered graph is Pfaffian. 
Corollary 3.11. The problem of determining whether or not a given orientation $D$ of a matching covered graph $G$ is Pfaffian is polynomially reducible to the problem of deciding whether or not a given matching covered graph is Pfaffian.

Proof. Determine whether $G$ is Pfaffian. If $G$ is not Pfaffian then certainly $D$ is not Pfaffian. Assume thus that $G$ is Pfaffian. Then, $p(H)=0$, for each conformal subgraph $H$ of $G$. Run the algorithm described in the proof of Lemma 3.8, in order to obtain an ear decomposition

$$
G_{1} \subset G_{2} \subset \ldots \subset G_{r}=G
$$

of $G$, where, for $i=2,3, \ldots, r, G_{i-1}=G_{i}-R_{i}$, and $R_{i}$ is a $b$-removable ear of $G$. For each $i, 1 \leq i \leq r$, let $D_{i}$ denote the restriction of $D$ to $G_{i}$.

For $i=1,2, \ldots, r$, we now determine, in polynomial time, whether $D_{i}$ is Pfaffian, and, if the answer is negative, a conformal circuit $Q$ of $G_{i}$ that has even parity in $G_{i}$. If $i=1$, then $D_{i}$ is certainly Pfaffian. Assume thus that $i>1$. If $D_{i-1}$ is non-Pfaffian, then let $Q$ be a conformal circuit of $G_{i-1}$ that has even parity in $D_{i-1}$. Then, $Q$ is a conformal circuit of $G_{i}$ that has even parity in $D_{i}$. Assume thus that $D_{i-1}$ is Pfaffian. In polynomial time, determine a conformal circuit of $G_{i}$ that contains some edge in the $b$-removable ear $R_{i}$ of $G_{i}$. If $Q_{i}$ has even parity in $D_{i}$ then $D_{i}$ is certainly non-Pfaffian. Assume thus that $Q_{i}$ has odd parity in $D_{i}$. By Corollary 3.6, it follows that $D_{i}$ is a Pfaffian orientation of $G_{i}$. In particular, the algorithm either determines that $D$ is Pfaffian, or alternatively, returns a conformal circuit of $G$ that has even parity in $D$.

\section{A Characterization of Pfaffian bipartite graphs}

The first results in this section provide the basis for the characterization of Pfaffian bipartite graphs. Recall that braces are bipartite matching covered graphs free of nontrivial tight cuts. Braces have an important property concerning removable edges.

Lemma 4.1 (see [2], Lem. 3.2). Let $G$ be a brace on at least six vertices. Then every edge of $G$ is removable.

The following lemma provides a characterization of non-removable edges in matching covered bipartite graphs.

Lemma 4.2 (see [12], Th. 4.1.1). Let $G[A, B]$ be a bipartite matching covered graph distinct from $K_{2}$ and let $e$ be an edge of $G$. Then, $e$ is not removable in $G$ if and only if there is a partition $\left\{A^{\prime}, A^{\prime \prime}\right\}$ of $A$ and a partition $\left\{B^{\prime}, B^{\prime \prime}\right\}$ of $B$ with $\left|A^{\prime}\right|=\left|B^{\prime}\right|$ such that $e$ is the only edge joining a vertex in $A^{\prime}$ to a vertex in $B^{\prime \prime}$.

Many properties that do not hold in general for bipartite matching covered graphs hold for bipartite graphs obtained from the deletion of a removable edge from a brace. We now describe some of those properties.

Let $G$ be a matching covered graph $G$ with bipartition $\{A, B\}$. For each shore $X$ of a tight cut $C$, the numbers $|X \cap A|$ and $|X \cap B|$ differ by exactly one, by 
Proposition 2.1. Whichever of $X \cap A$ and $X \cap B$ is the largest, it is called the majority part of $X$, whereas the other is the minority part of $X$. The next property is immediate from the fact that braces are free of tight cuts and Proposition 2.1.

Proposition 4.3. Let $G$ be a brace, e a removable edge of $G, C:=\partial(X)-e$ a nontrivial tight cut of $G-e$. Then, $e$ is the only edge of $G$ that has its ends in the minority parts of $X$ and of $\bar{X}$.

Lemma 4.4. Let $G$ be a brace, e a removable edge of $G, C:=\partial(X)-e$ a nontrivial tight cut of $G-e$. Then, each edge of $C$ is removable in $G-e$.

Proof. Assume to the contrary that there is an edge $e^{\prime}$ in $C$ that is not removable in $G-e$. Then, $e^{\prime}$ is not removable in some (bipartite) $C$-contraction of $G-e$, by Proposition 2.2. Adjust notation so that $e^{\prime}$ is not removable in the $C$-contraction $H$ obtained from $G$ by contracting $\bar{X}$ to a single vertex $\bar{x}$. Let $\{A, B\}$ denote the bipartition of $H$. Adjust notation so that $\bar{x}$ lies in $A$.

Let $y$ denote the end of $e^{\prime}$ in $\bar{X}$. Thus, $y$ lies in the majority part of $\bar{X}$. By Lemma 4.2, there exists a partition $\left(B^{\prime}, B^{\prime \prime}\right)$ of $B$ and a partition $\left(A^{\prime}, A^{\prime \prime}\right)$ of $A$ such that $\left|A^{\prime}\right|=\left|B^{\prime}\right|$ and $e^{\prime}$ is the only edge of $H$ that joins a vertex of $A^{\prime}$ to a vertex of $B^{\prime \prime}$. Then, $Y:=A^{\prime \prime} \cup B^{\prime \prime} \cup\{y\}$ is the shore of a nontrivial tight cut of $G-e$. Moreover, edge $e$ has one end in $\bar{X}$, the other in $X \cap A$, the minority part of $X$. Thus, no end of $e$ lies in $B^{\prime \prime}$. We conclude that $Y$ is the shore of a nontrivial tight cut of $G$, a contradiction. As asserted, every edge of $C$ is removable in $G-e$.

Suppose that $G$ is a brace and $e$ is a removable edge of $G$. It turns out that one can always find at least two removable edges of $G-e$ incident with any vertex, provided that vertex is of degree greater than two in $G-e$, as shown in the next result.

Lemma 4.5. Let $G[A, B]$ be a brace, e a removable edge of $G$, $v$ any vertex of $G$. If the degree of $v$ is greater than two in $G-e$ then at most one edge of $G-e$ incident with $v$ is not removable in $G-e$.

Proof. If every edge of $G-e$ incident with $v$ is removable in $G-e$ then the assertion holds immediately. We may thus assume that there is an edge $e^{\prime}$ incident with $v$ that is not removable in $G-e$. We must now show that every edge of $\partial(v)-e-e^{\prime}$ is removable in $G-e$.

By Lemma 4.2 , there is a partition $\left\{A^{\prime}, A^{\prime \prime}\right\}$ of $A$ and a partition $\left\{B^{\prime}, B^{\prime \prime}\right\}$ of $B$ with $\left|A^{\prime}\right|=\left|B^{\prime}\right|$ such that $e^{\prime}$ is the only edge of $G-e$ joining a vertex in $A^{\prime}$ to a vertex in $B^{\prime \prime}$. Assume, without lost of generality, that $v \in A^{\prime}$. Then $X^{\prime}:=\left(A^{\prime}-v\right) \cup B^{\prime}$ is the shore of a (possibly trivial) tight cut $C^{\prime}$ of $G-e$.

Consider first the case in which $X^{\prime}$ is a singleton. In that case, each edge of $\partial(v)-e-e^{\prime}$ joins $v$ to the only vertex of $X^{\prime}$. By hypothesis, the degree of $v$ in $G-e$ is three or more. Therefore, the edges of $\partial(v)-e-e^{\prime}$ are multiple edges. We conclude that the assertion holds in this case.

We may thus assume that $X^{\prime}$ is not a singleton. As $\overline{X^{\prime}}$ contains all the vertices of $B^{\prime \prime}$ and also vertex $v$, it follows that $\overline{X^{\prime}}$ is not a singleton either. Thus, $C^{\prime}$ is a 
nontrivial tight cut of $G-e$. Moreover, all the edges of $\partial(v)-e-e^{\prime}$ lie in $C^{\prime}$. By Lemma 4.4, each edge of $C^{\prime}$ is removable in $G-e$. Thus, each edge of $\partial(v)-e-e^{\prime}$ is removable in $G-e$.

Theorem 4.6. A bipartite matching covered graph $G$ is Pfaffian if and only if it does not contain $K_{3,3}$ as a minor.

Proof. If $G$ contains $K_{3,3}$ as a minor then clearly $G$ is non-Pfaffian, for $K_{3,3}$ is non-Pfaffian. Now, suppose that $G$ is non-Pfaffian, and let us show, by induction, that $G$ contains $K_{3,3}$ as a minor.

If $G$ contains a proper minor $H$ that is non-Pfaffian then, by induction hypothesis, $H$ contains $K_{3,3}$ as a minor. As the minor property is transitive, $G$ also contains $K_{3,3}$ as a minor. We may thus assume that all proper minors of $G$ are Pfaffian. We shall prove that $G$ is $K_{3,3}$. As $G$ is free of non-Pfaffian proper minors, it follows that $G$ is simple. Graphs $K_{2}$ and $C_{4}$ are the only simple bipartite matching covered graphs on two and four vertices, respectively, and they are Pfaffian graphs. It follows that $G$ contains at least six vertices.

Proposition 4.7. Graph $G$ is a brace.

Proof. Observe first that every (not necessarly removable) single ear of $G$ has length one, otherwise, $G$ is an even subdivision of a bipartite graph $H$ that is non-Pfaffian and a proper minor of $G$, a contradiction.

We now prove that $G$ is free of vertices of degree two. For this, assume, to the contrary, that $G$ has a vertex $v$, that has precisely two neighbors, $u$ and $w$. Let $X:=\{u, v, w\}, C:=\partial(X)$. As $\mid V(G) \geq 6, C$ is a nontrivial tight cut of $G$. The $C$-contraction $G^{\prime}$ of $G$ that contains the vertices in $X$ is $C_{4}$, up to multiple edges, whence it is Pfaffian. By Theorem 2.4, the other $C$-contraction of $G$, denoted $G^{\prime \prime}$, is non-Pfaffian. Vertices $u$ and $w$ both have degree grater than two in $G$, because $G$ is free of single ears of length greater than one. Thus, the contraction vertex, say $x$, of $G^{\prime \prime}$ has degree at least four in $G^{\prime \prime}$. Moreover, each edge of $C$ is a multiple edge in $G^{\prime}$. By induction hypothesis, $G^{\prime \prime}$ has $K_{3,3}$ as a minor. As $K_{3,3}$ is cubic and $x$ has degree four in $G^{\prime \prime}$, it follows that $K_{3,3}$ is a proper minor of $G^{\prime \prime}$. As $G$ is free of single ears of length greater than one and $x$ has degree four in $G^{\prime \prime}$, it follows that $G^{\prime \prime}$ is also free of single ears of length greater than one. We conclude that $G^{\prime \prime}$ has a removable edge $e$ such that $G^{\prime \prime}-e$ is non-Pfaffian. If $e$ does not lie in $C$ then $e$ is removable in $G$. If $e$ lies in $C$ then it is a multiple edge in $G^{\prime}$, whence removable in $G^{\prime}$. In both alternatives we conclude that $e$ is removable in $G$, by Proposition 2.2. Clearly, $C-e$ is a tight cut of $G-e$ and one the $(C-e)$ contractions of $G-e$ is $G^{\prime \prime}-e$, in turn a non-Pfaffian graph. By Theorem 2.4, $G-e$ is non-Pfaffian. This is a contradiction to the hypothesis that $G$ is free of proper non-Pfaffian minors. As asserted, $G$ is free of vertices of degree two.

Finally, assume, to the contrary, that $G$ has a non-trivial tight cut $C$. By Theorem 2.4, at least one of the $C$-contractions of $G$ is non-Pfaffian. Let $X$ be a minimal subset of $V(G)$ such that $C:=\partial(X)$ is a nontrivial tight cut of $G$ and the $C$-contraction $G^{\prime}$ of $G$, obtained from $G$ by contracting $X$ to a single vertex $x$, is non-Pfaffian. Note that the other $C$-contraction of $G$, denoted $G^{\prime \prime}$, must be a 
brace, by the minimality of $X$, and by Theorem 2.4 and Proposition 2.1. Let $v$ be any vertex of $X$ in the minority part of $G^{\prime \prime}$. Then, no edge of $\partial(v)$ lies in $C$. Vertex $v$ must be adjacent to at least three vertices of $G$, because $G$ is simple and free of vertices of degree two. It follows that $G^{\prime \prime}$ is a brace with at least six vertices. Let $e$ be any edge of $\partial(v)$. By Lemma 4.1, $e$ is removable in $G^{\prime \prime}$. As $e$ does not lie in $C$, it is removable in $G$. Cut $C$ is a tight cut of $G-e$, the $C$-contraction $G^{\prime}$ of $G-e$ is non-Pfaffian. By Theorem 2.4, $G-e$ is non-Pfaffian. Again, we have a contradiction to the hypothesis that $G$ is free of proper non-Pfaffian minors. We conclude that $G$ is free of nontrivial tight cuts. That is, $G$ is a brace.

Let $e$ be an edge of $G$. By Lemma 4.1, $G-e$ is matching covered and so, $G-e$ is Pfaffian. Consider a Pfaffian orientation $D$ of $G-e$. Then, every perfect matching of $G-e$ has the same sign relative to the given orientation.

Orient edge $e$ arbitrarily and consider the set $\mathcal{M}_{e}$ of all perfect matchings of $G$ containing $e$. The matchings in $\mathcal{M}_{e}$ cannot have the same sign, otherwise, by possibly changing the orientation of $e$, we get all perfect matchings of $G$ with the same sign and we conclude that $G$ is Pfaffian, a contradiction. Then, there exist two perfect matchings $F_{1}$ and $F_{2}$ in $\mathcal{M}_{e}$ with distinct signs. We now determine properties of matchings $F_{1}$ and $F_{2}$.

Proposition 4.8. Every removable edge of $G-e$ lies in $F_{1} \cup F_{2}$.

Proof. Suppose that $G-e$ contains a removable edge $f$ that does not lie in $F_{1} \cup F_{2}$. Then, $G-e-f$ is a bipartite matching covered graph. Moreover, $D-f$ is a Pfaffian orientation of $G-e-f$. As $e$ has its ends in distinct parts of $G-e-f$, addition of $e$ to $G-e-f$ produces a matching covered graph (see [12, Th. 4.1.1]). Thus, $G-f$ is matching covered.

As all proper minors of $G$ are Pfaffian, $G-f$ is Pfaffian. As $D-f$ is a Pfaffian orientation of $G-e-f$, then, by Corollary 3.6, $D-f$ has an extension $D^{\prime}$ to an orientation of $G-f$ that is Pfaffian. But $F_{1}$ and $F_{2}$ are perfect matchings of $G-f$ having distinct signs in $D^{\prime}$. This is a contradiction. Thus, every removable edge of $G-e$ lies in $F_{1} \cup F_{2}$.

Proposition 4.9. The set $F_{1} \cap F_{2}-e$ is empty.

Proof. Assume, to the contrary, that $F_{1} \cap F_{2}-e$ contains an edge, say $f$. Let $v$ denote an end of $f$. As $f$ and $e$ are distinct edges of a matching, they are nonadjacent. Thus, $v$ is not an end of $e$. As $G$ is a brace, vertex $v$ has degree at least three in $G-e$. By Lemma 4.5, $\partial(v)-f$ contains an edge that is removable in $G-e$. That edge does not lie in $F_{1} \cup F_{2}$, a contradiction to Proposition 4.8.

By Theorem 1.1, $F_{1} \oplus F_{2}$ contains a circuit $C$ of even parity. Moreover, $e$ does not lie in $C$. Let $F_{2}^{\prime}:=F_{1} \oplus C$. Then, $F_{1}$ and $F_{2}^{\prime}$ are perfect matchings of $G$ with distinct parities, both of which contain edge $e$. If $E(C)$ is a proper subset of $F_{1} \oplus F_{2}$, then $F_{1} \cap F_{2}^{\prime}-e$ is nonnull, in contradiction to Proposition 4.9. We conclude that $E(C)=F_{1} \oplus F_{2}$. Thus, $C$ is a Hamiltonian circuit of $G-u-v$, where $u$ and $v$ are the ends of $e$. 
Now, suppose that $C$ contains a chord $f$. Then, $C+f$ is the union of two circuits of $G$ that do not contain $e$. Moreover, as $G$ is simple and bipartite, both circuits have sizes smaller than $C$, one of them, say $C_{1}$, is $F_{1}$-alternating, the other, $C_{2}$, is $F_{2}$-alternating. As $C$ has even parity, one of $C_{1}$ and $C_{2}$, say $C_{2}$, has even parity. Then, $F_{2}^{\prime}:=F_{2} \oplus C_{2}$ is a perfect matching of $G$ that contains edge $e$, has sign distinct from that of $F_{1}$ and $F_{1} \cap F_{2}^{\prime}-e$ is nonnull, a contradiction. Thus, $C$ does not contain a chord. That is, $G-u-v=C$.

As $G$ is a brace, every vertex of $G$ has degree at least three. As $G$ is bipartite, every vertex of $C$ must be adjacent to precisely one of $u$ and $v$. By Proposition 4.8 and Lemma 4.5, the ends of $e$ must have degree three in $G$. We conclude that $C=C_{4}$ and $G=K_{3,3}$.

\section{Final REMARKS}

As mentioned in the introduction, the Pfaffian orientation problem has been completely solved for planar graphs and bipartite graphs. Generalizing Little's theorem, Fischer and Little [7] found an excluded conformal minor characterization of near-bipartite graphs. Apart from these results, as far as we know, the complexity status of the Pfaffian orientation problem is very much open.

A cut $C$ in a matching covered graph is separating if both $C$-contractions are matching covered. Thus, tight cuts are separating cuts. But, in general, a separating cut need not be a tight cut. We say that a brick is solid if it is free of non-trivial separating cuts. Solid bricks are precisely the bricks whose perfect matching polytopes are described in terms of degree constraints and non-negativity [1].

Conjecture 5.1. A solid brick is Pfaffian if and only if it does not have a minor having $K_{3,3}$ as a brace.

Note added in proof: Since writing this paper, we have been able to prove Conjecture 5.1.

\section{REFERENCES}

[1] M.H. de Carvalho, C.L. Lucchesi and U.S.R. Murty, The perfect matching polytope and solid bricks. J. Combin. Theory B 92 (2004) 319-324.

[2] M.H. de Carvalho, C.L. Lucchesi and U.S.R. Murty, Ear decompositions of matching covered graphs. Combinatorica 19 (1999) 151-174.

[3] M.H. de Carvalho, C.L. Lucchesi and U.S.R. Murty, On a conjecture of Lovász concerning bricks. I. The characteristic of a matching covered graph. J. Comb. Theory B $\mathbf{8 5}$ (2002) 94-136.

[4] M.H. de Carvalho, C.L. Lucchesi and U.S.R. Murty, On a conjecture of Lovász concerning bricks. II. Bricks of finite characteristic. J. Comb. Theory B 85 (2002) 137-180.

[5] M.H. de Carvalho, C.L. Lucchesi and U.S.R. Murty, Optimal ear decompositions of matching covered graphs. J. Comb. Theory B $\mathbf{8 5}$ (2002) 59-93.

[6] J. Edmonds, L. Lovász and W.R. PulleyblanK, Brick decomposition and the matching rank of graphs. Combinatorica 2 (1982) 247-274.

[7] I. Fischer and C.H.C. Little, A characterisation of Pfaffian near bipartite graphs. J. Comb. Theory B 82 (2001) 175-222. 
[8] P.W. Kasteleyn, Dimer statistics and phase transitions. J. Math. Phys. 4 (1963) 287-293.

[9] C. Little, A characterization of convertible (0,1)-matrices. J. Comb. Theory B 18 (1975) $187-208$.

[10] C.H.C. Little and F. Rendl, Operations preserving the Pfaffian property of a graph. J. Austral. Math. Soc. Ser. A 50 (1991) 248-275.

[11] L. Lovász, Matching structure and the matching lattice. J. Comb. Theory B 43 (1987) $187-222$.

[12] L. Lovász and M.D. Plummer, Matching Theory. Annals of Discrete Mathematics, vol. 29. Elsevier Science (1986).

[13] W. McCuaig, Brace generation. J. Graph Theory 38 (2001) 124-169.

[14] N. Robertson, P.D. Seymour and R. Thomas, Permanents, Pfaffian orientations and even directed circuits. Ann. Math. 150 (1999) 929-975.

[15] W.T. Tutte, Graph Theory as I Have Known It. Number 11 in Oxford Lecture Ser. Math. Appl. Clarendon Press, Oxford (1998).

[16] V.V. Vazirani and M. Yanakakis, Pfaffian orientation of graphs, 0,1 permanents, and even cycles in digraphs. Discrete Appl. Math. 25 (1989) 179-180. 